\title{
In Vivo Determination of the Complex Elastic Moduli of Cetacean Head Tissue
}

\author{
Peter H. Rogers \& Michael D. Gray \\ G. W. Woodruff School of Mechanical Engineering \\ Georgia Institute of Technology \\ Atlanta, GA 30332 \\ phone: (404) 894-3235 fax: (404) 894-7790 email: peter.rogers@me.gatech.edu \\ phone: (404) 385-1872 fax: (404) 894-7790 email: michael.gray@me.gatech.edu
}

Award Number: N00014-08-1-1158

\section{LONG-TERM GOALS}

The overall goal of this project is to develop and demonstrate a system for non-invasive in vivo measurement of the complex elastic moduli (stiffnesses and loss factors) of cetacean head soft tissues. This system is ultimately intended to provide a portable diagnostic capability for use in stranded animal assessments.

\section{OBJECTIVES}

The primary objective of this project is to develop an ultrasound-based system for non-invasive determination of in vivo shear and bulk properties of cetacean soft tissues, including jaw fats and brain. The ultimate goal is to field a prototype system for examinations of stranded animals. Data collected with this system is intended to provide: 1) basic knowledge of in vivo tissue viscoelastic properties, and 2) a potential basis for diagnostics of tissue pathologies.

\section{APPROACH}

This work builds upon the principles of ultrasonic elastography ${ }^{\mathrm{i}}$, wherein ultrasound is used to both generate and observe low frequency vibration in soft tissues. While current methods have been successfully applied for human subjects, they are limited to tissue depths in the range of $5 \mathrm{~cm}$, and cannot be directly extended deeper or through bone while remaining in compliance with federally mandated safety restrictions for ultrasound exposure ${ }^{\text {ii }}$.

The focus of the present research was to overcome these limitations through reconsideration of the methods by which wave motion in soft tissues is generated and measured. The measurement concept thus developed, called convergent field elastography (CFE), uses a specially shaped ultrasonic field to safely generate larger soft tissue displacements at extended depths. The CFE concept is illustrated in Figure 1. Forces are remotely generated inside soft tissues using an ultrasonic transducer that produces a tube-like intensity pattern. The response to this force is primarily observable as an inwardly propagating shear wave field that grows in amplitude as it converges to the center of the force pattern. A second ultrasonic transducer monitors the tissue displacement along the ultrasound beam axis. This 


\section{Report Documentation Page}

Form Approved

OMB No. 0704-0188

Public reporting burden for the collection of information is estimated to average 1 hour per response, including the time for reviewing instructions, searching existing data sources, gathering and maintaining the data needed, and completing and reviewing the collection of information. Send comments regarding this burden estimate or any other aspect of this collection of information,

including suggestions for reducing this burden, to Washington Headquarters Services, Directorate for Information Operations and Reports, 1215 Jefferson Davis Highway, Suite 1204, Arlington

VA 22202-4302. Respondents should be aware that notwithstanding any other provision of law, no person shall be subject to a penalty for failing to comply with a collection of information if it

does not display a currently valid OMB control number.

1. REPORT DATE

30 SEP 2014

4. TITLE AND SUBTITLE

In Vivo Determination of the Complex Elastic Moduli of Cetacean Head Tissue

6. $\operatorname{AUTHOR}(\mathrm{S})$

7. PERFORMING ORGANIZATION NAME(S) AND ADDRESS(ES)

Georgia Institute of Technology,School of Mechanical Engineering,Atlanta,GA,30332

9. SPONSORING/MONITORING AGENCY NAME(S) AND ADDRESS(ES)

3. DATES COVERED

00-00-2014 to 00-00-2014

5a. CONTRACT NUMBER

5b. GRANT NUMBER

5c. PROGRAM ELEMENT NUMBER

5d. PROJECT NUMBER

5e. TASK NUMBER

5f. WORK UNIT NUMBER

8. PERFORMING ORGANIZATION REPORT NUMBER

10. SPONSOR/MONITOR'S ACRONYM(S)

11. SPONSOR/MONITOR'S REPORT NUMBER(S)

12. DISTRIBUTION/AVAILABILITY STATEMENT

Approved for public release; distribution unlimited

13. SUPPLEMENTARY NOTES

14. ABSTRACT

15. SUBJECT TERMS

16. SECURITY CLASSIFICATION OF:

a. REPORT

unclassified b. ABSTRACT unclassified c. THIS PAGE

unclassified
17. LIMITATION OF ABSTRACT

Same as

Report (SAR)
18. NUMBER 19a. NAME OF

OF PAGES RESPONSIBLE PERSON

13 
inner transducer supports an enhanced embodiment of an ultrasonic vibrometry concept (non invasive vibration measurement system - NIVMS) previously developed at Georgia Tech.

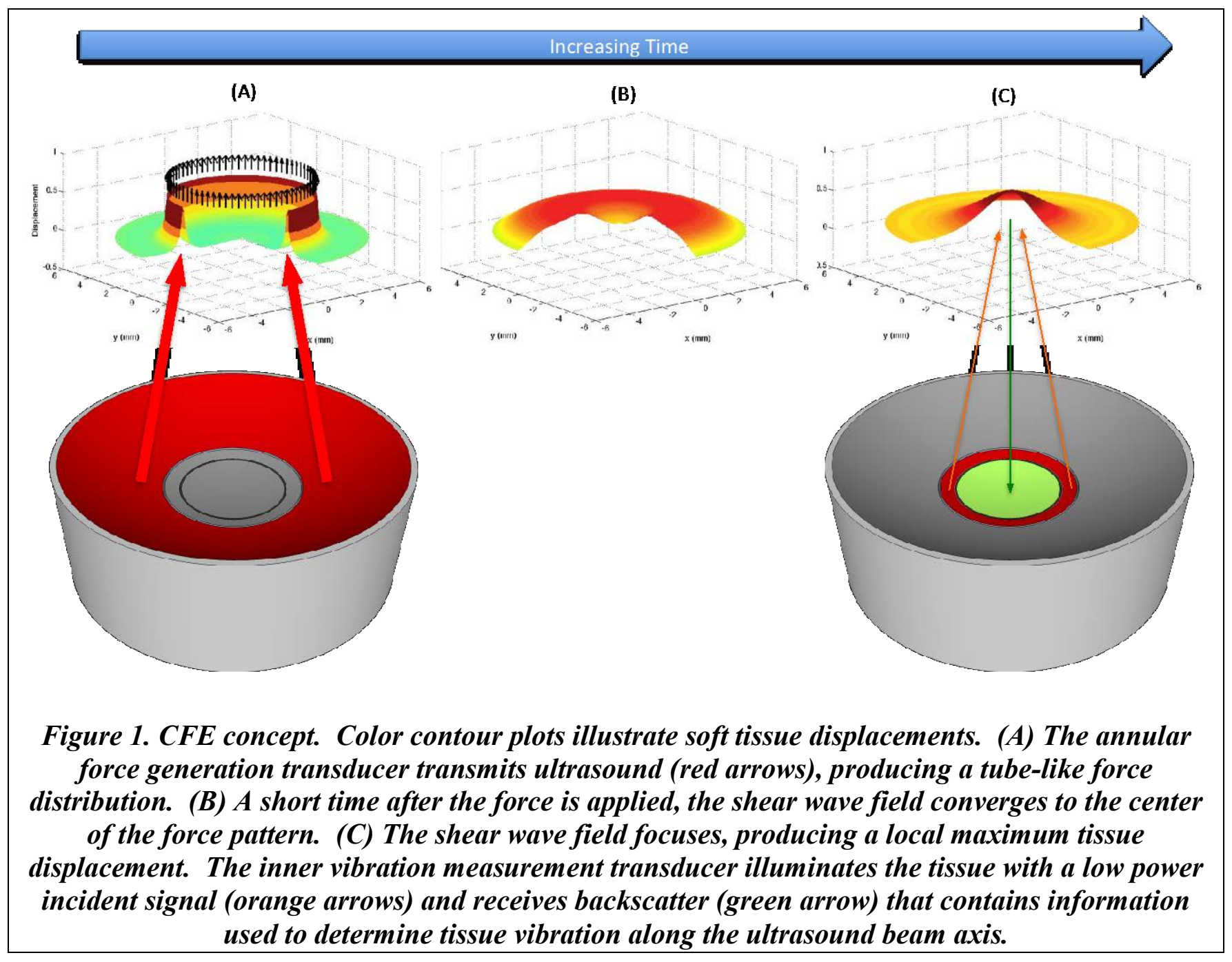

Soft tissue shear tissue properties are determined through control of the mean radius and modulation rate of the forcing field. Variation of mean radius provides information on propagation delay, which is used to estimate shear wave speed at a single frequency. By modulating the forcing beam at a controlled rate, the frequency dependence of shear speed can be determined, and the shear loss subsequently found through a model fit to the observed dispersion.

The CFE system was designed through simulation, and a prototype was constructed for concept demonstration. Evaluation of the prototype was conducted with tissue phantoms, at first with homogeneous construction and subsequently with spherical inclusions of contrasting shear stiffness. A subset of the phantom testing will be repeated with samples of cetacean mandibular and cranial bone obscuring the ultrasonic beam paths. Successful completion of lab experiments will be followed as funding allows by in vivo testing on Navy dolphins and stranded animals, for which permits and approvals have been obtained. 


\section{WORK COMPLETED}

\section{$\underline{\text { System Design }}$}

A prototype CFE system was designed based on combined simulations of ultrasonic field formation and viscoelastic tissue response. A design goal of $14 \mathrm{~cm}$ was chosen for the prototype system's focal length. This would allow examination of brain tissue in a typical adult bottlenose dolphin, which is the target for initial in vivo testing. Specifications for the prototype force generation and vibration measurement transducers were developed, and the transducers were constructed by a custom ultrasonic equipment manufacturer.

\section{Transducer Evaluation}

The prototype transducers were calibrated after receipt from the manufacturer and compared with the performance expected based on design simulations. This process occurred several times due to the need to repair leaks.

\section{Phantom Development}

Tissue mimicking phantoms were developed in concert with two manufacturers in order to produce laboratory test subjects whose properties were representative of mammalian muscle and brain tissues. Internal scattering levels were adjusted to match those observed in preliminary in vivo experiments (conducted under a separate effort) on two bottlenose dolphins and one beluga whale.

\section{System Testing}

The CFE prototype was tested on a phantom with properties representative of muscle tissue. An essential part of preparing the system for use was identification and suppression of coupling between the force generation and vibrometer transducers. This coupling appears in the processed system output as false signals that obscure the underlying vibration data. Suppression of the coupling effects was implemented through a combination of pre- and post-processing methods that were first validated through experimentation with a point-like target whose motion was predicted and independently measured with a laser vibrometer. Further revisions to the techniques were made to compensate for distributed scattering effects present in tissue phantoms.

\section{RESULTS}

\section{$\underline{\text { System Development }}$}

Numerical simulations were carried out in order to assess the relative merits of candidate transducer designs and to gain a preliminary understanding of the expected performance of the CFE concept. For the force generation transducer, a relatively simple means was sought for generating a beam pattern with an on-axis minimum, and with primary lobes lying on a radius of 2-4 millimeters. This range of radii was based on considerations of thermal effects (how closely can multiple beams be spaced without significant thermal "communication") and nearfield effects from the force distribution (the change in phase with respect to distance is not simply characterized by $k_{s} \Delta r$ in the extreme nearfield of the force). Several force generation transducer configurations were considered, but ultimately a sectored, spherically focused design, illustrated in Figure 2, was chosen for its relative simplicity both in design and drive requirements. 

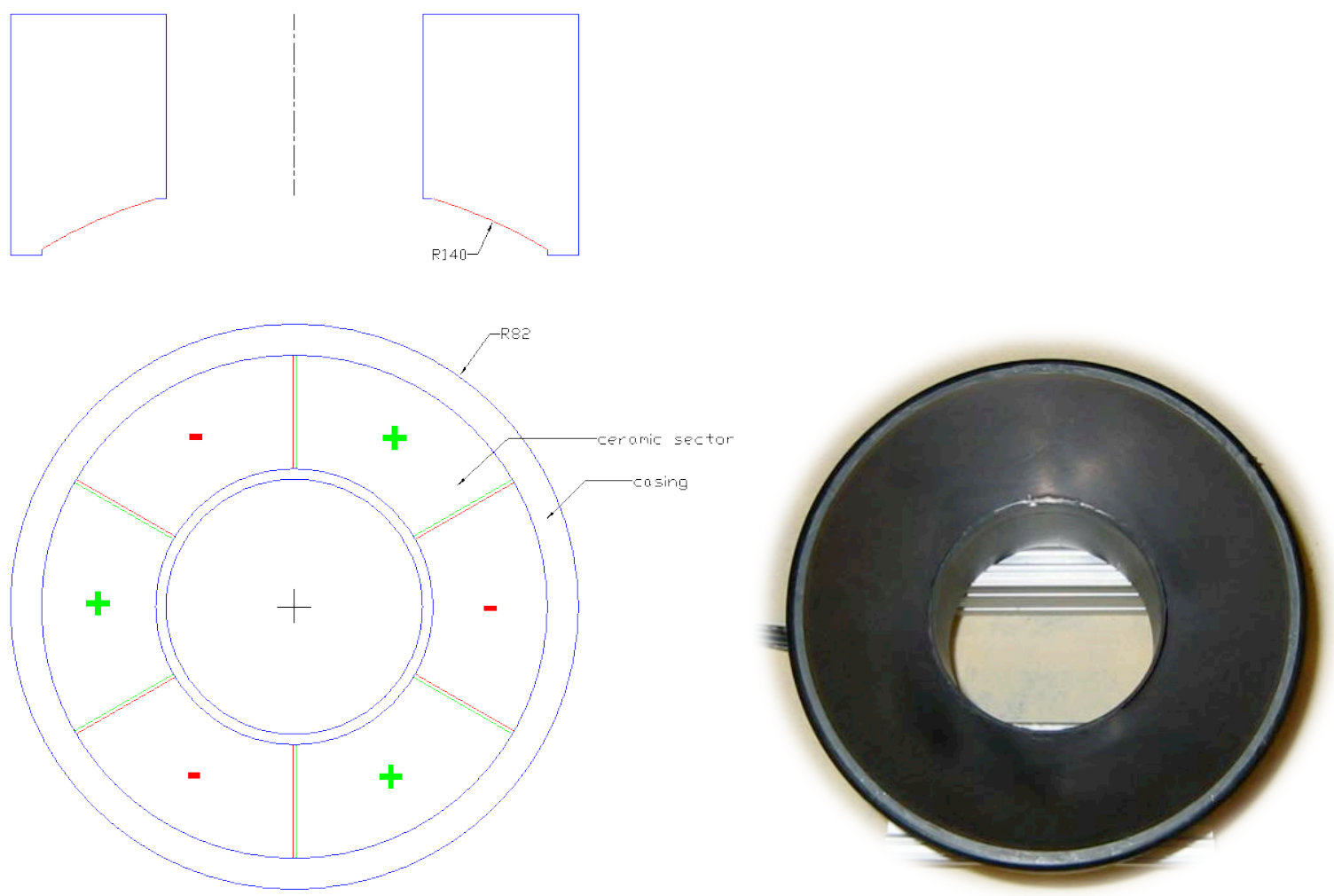

Figure 2. Design (left) and fabrication (right) of the ultrasound force generation transducer. Dimensions in mm.

Figure 3 shows example predicted focal plane field patterns in water for a single carrier frequency, along with measurements made on the manufactured prototype. The desired beam pattern was arrived at by driving adjoining sectors with identical signals but with opposite phase (see +/- symbols in Fig. 2). Since the transducer volume velocity has a zero mean value, there is no on-axis main lobe at the geometric focal distance, and the focal plane field is characterized by a set of lobes uniformly offset from the array's symmetry axis. The number of lobes corresponds to the number of sectors, and the location and size of the lobes is controlled through by transducer geometry and drive frequency. Specifically, the radius to and width of each lobe increases inversely with frequency.

The patterns in Figure 3 are normalized by the focal plane maxima. The transducer produces a field pattern with a center null and six lobes distributed on a single radius. Such a pattern would produce six simultaneous forcing regions, which may be thought of as a discretized representation of a forcing ring. The critical aspect of the sectored design was achieved - namely that the on-axis field was minimized without any weighting of the individual elements other than signal polarity. As seen in Figure 4 , the ability to shift the pattern radius with carrier frequency was as expected. The actual radius on which the primary lobes appeared was slightly smaller in the prototype than in the prediction, but this is of no consequence to system operation. 

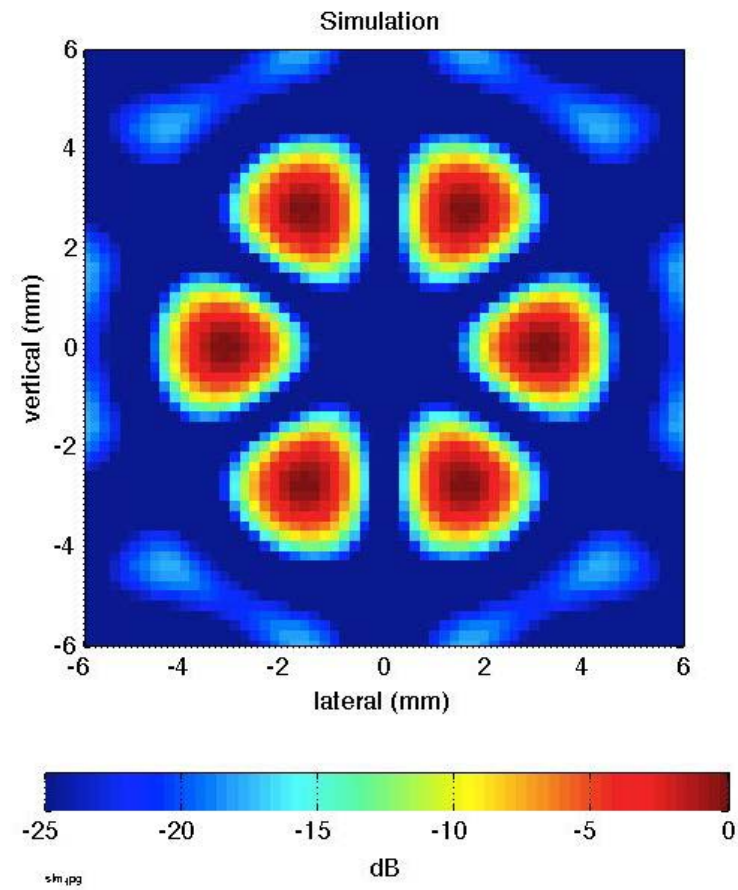
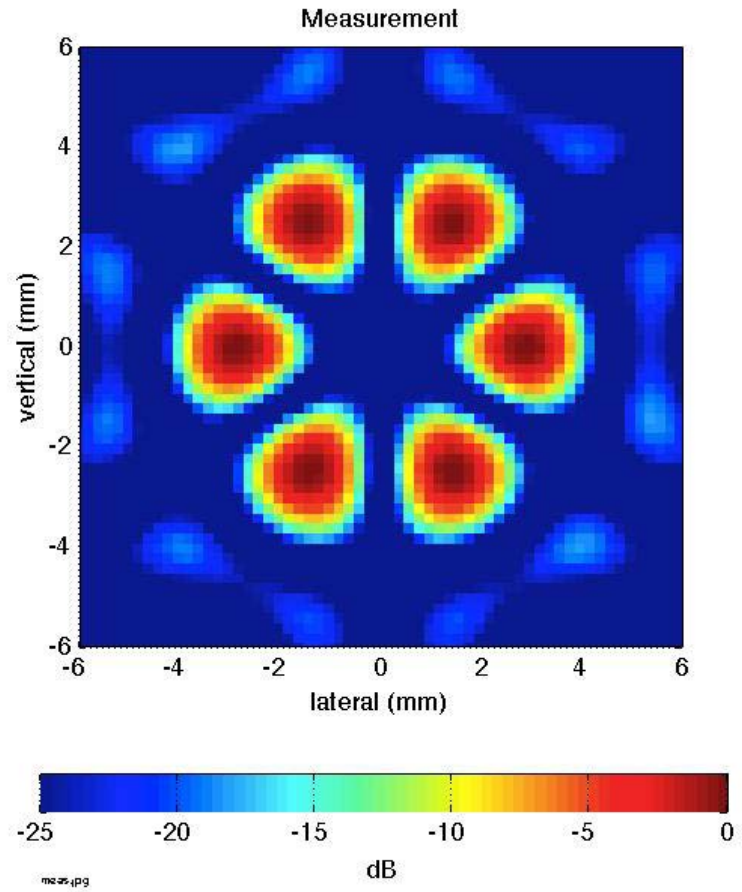

Figure 3. Predicted (left) and measured (right) focal plane pressures at $800 \mathrm{kHz}$.
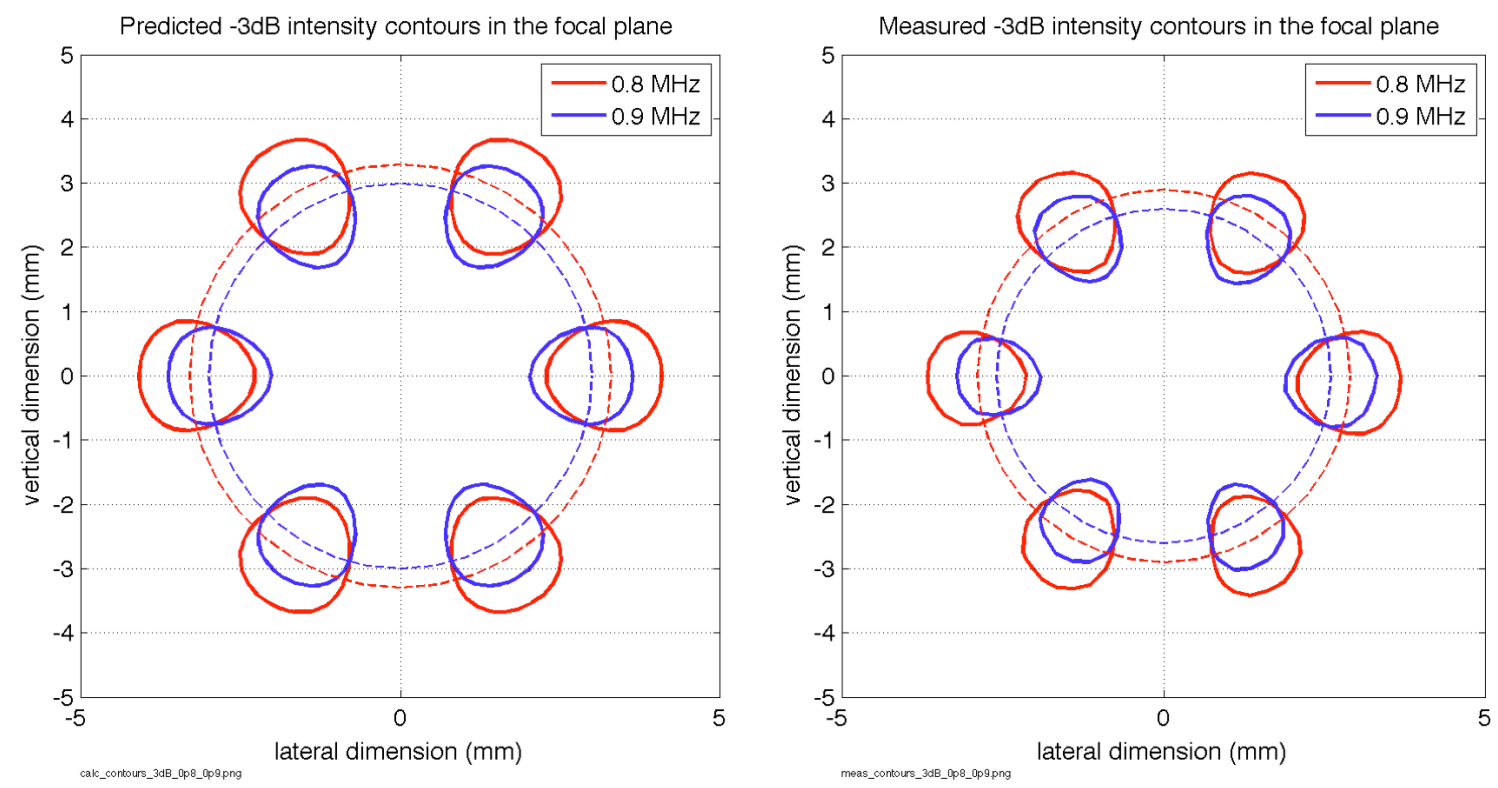

Figure 4. Predicted (left) and measured (right) intensity contours at 800 and $900 \mathrm{kHz}$. Dashed lines indicate lobe centroid radii. 
The vibrometer transducer, illustrated in Figure 5, employs a pair of confocal elements - one each for signal transmission and reception - designed for operation between 1 and $3 \mathrm{MHz}$. The dimensions of the elements were chosen so that the transmit-receive product pattern would have minimal sidelobes, particularly in the regions where the force generation beam amplitudes were largest. The housing was sized to give $2 \mathrm{~mm}$ of clearance with respect to the force generation transducer inner diameter. Figure 6 shows the predicted and measured on-axis responses of the vibrometer transducer. Noteworthy is the reduced axial field strength of the prototype in front of the focus. This was thought to be due to a higher than expected variation in the ceramic radius of curvature. The construction flaw (confirmed by the manufacturer) reduces the strength of the axial diffraction lobe - a result which is beneficial for the proposed application.
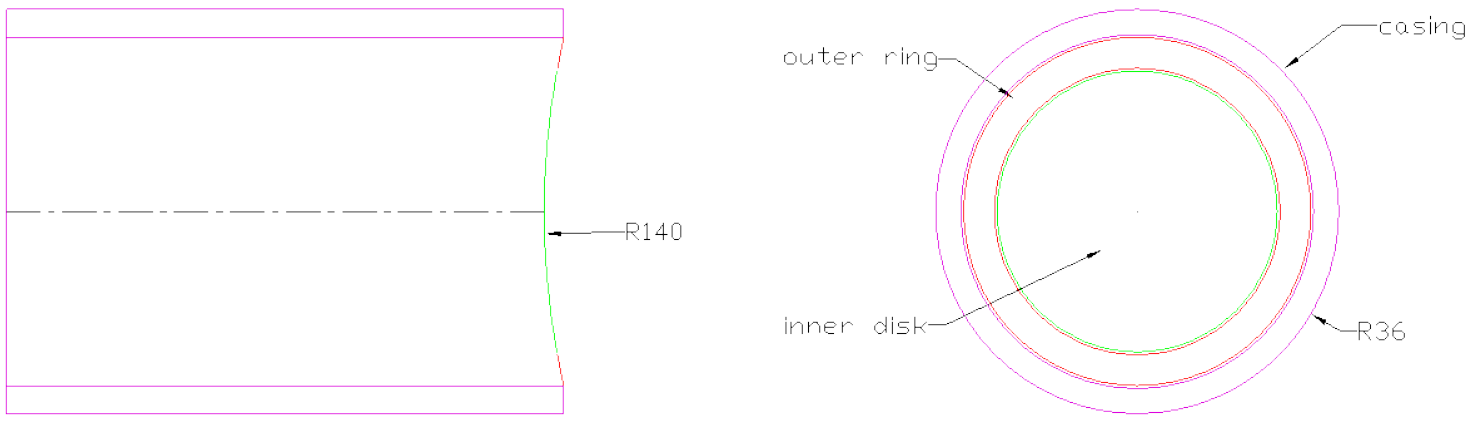

Figure 5. Vibrometer transducer design, dimensions in millimeters

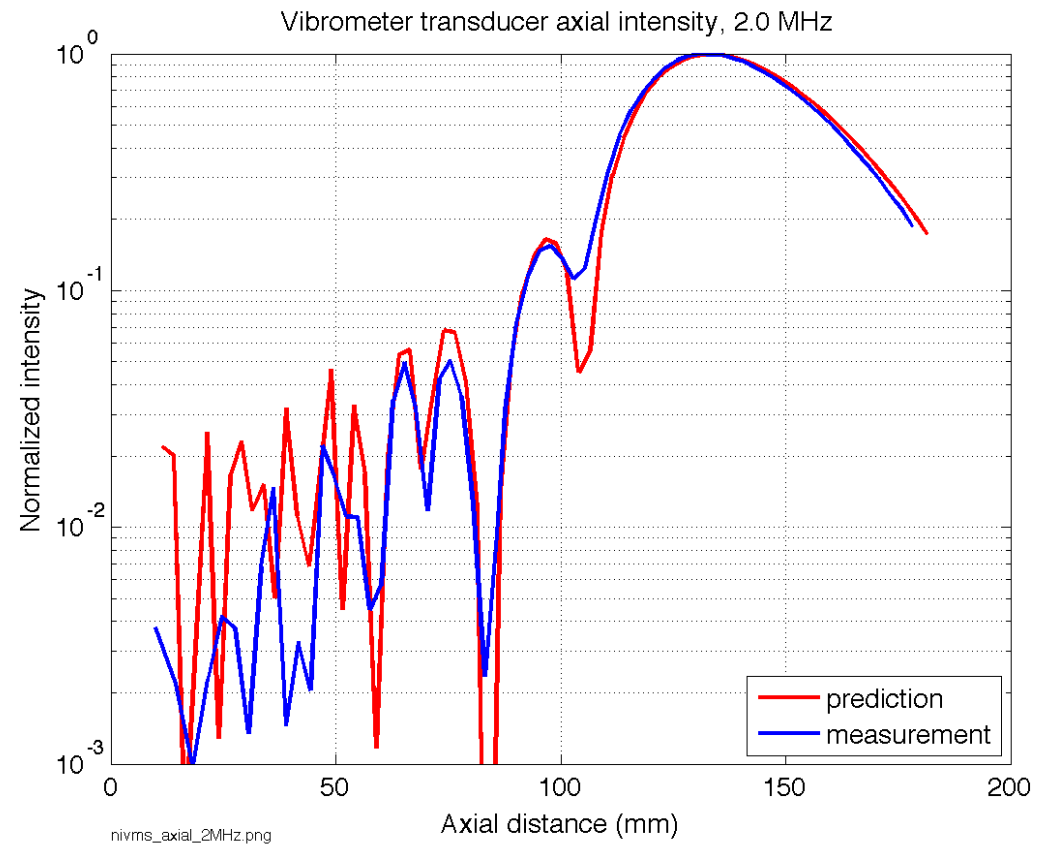

Figure 6. Predicted and measured vibrometer transducer axial field responses at $2 \mathrm{MHz}$ 

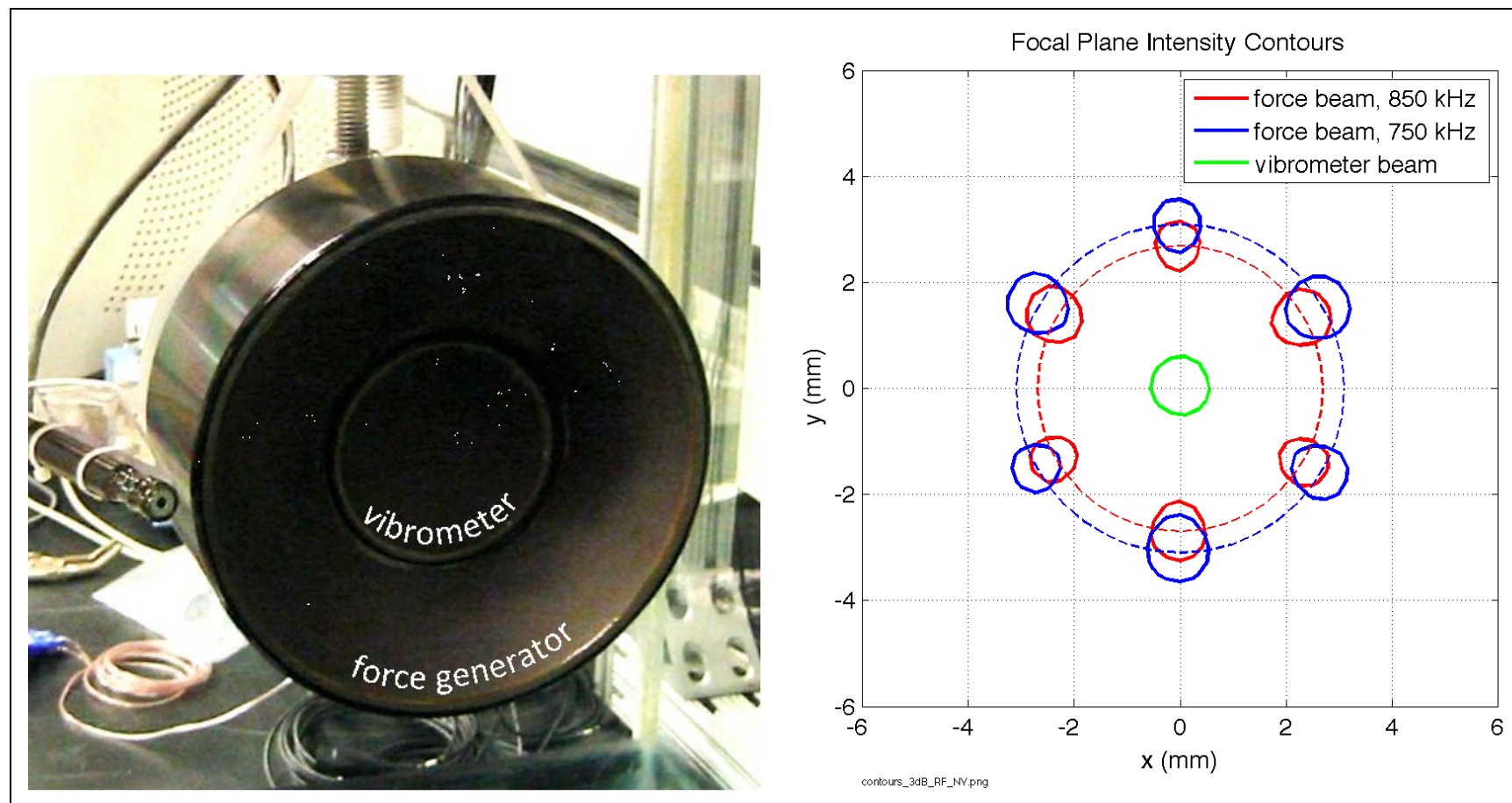

Figure 7. Assembled CFE prototype system (left), and its focal plane intensity contours (right).

Figure 7 shows the assembled CFE prototype, with the vibrometer nested inside the force generator. The two transducers are held together with a back plate that facilitates fine scale alignment of the transducer beams. The force generation and vibrometry beams are also nested, with forces applied around a perimeter, and the vibrometer beam monitoring shear fields converging to the center of the force pattern. Since all system beams are coaxial, it is anticipated that refraction effects in complex tissues will have a minimal impact on system performance.

\section{$\underline{\text { System Testing }}$}

For initial tissue phantom testing, the CFE system used the drive signals shown in Figure 8 . The force generation (RF) signal is a $2.92 \mathrm{~ms}$ duration Hanning windowed sequence of tone bursts, with duration and spacing chosen so as not to have temporal overlap with the vibrometer transmission (NV) or the backscatter of the NV signal from a depth of $12-14 \mathrm{~cm}$. The periodicity of the NV transmissions was $500 \mu \mathrm{s}$, so that the output sampling rate of the vibrometer system was $2 \mathrm{kHz}$.

To minimize the possibility of vibrometer aliasing, the RF signal duration and window shape were chosen to produce a low pass filtering effect, cutting off the drive signal at $1 \mathrm{kHz}$. The RF pulse repetition rate of $64 \mathrm{~Hz}$ was also chosen so that signal content above $1 \mathrm{kHz}$ would not alias onto signal content below $1 \mathrm{kHz}$ (i.e. the first tone above $1 \mathrm{kHz}$ does not wrap around to the last tone below $1 \mathrm{kHz}$ ). When processed as an ensemble of forcing pulses, the low frequency drive spectrum has a low pass filtered comb spectrum consisting of $64 \mathrm{~Hz}$ harmonics, as shown in the bottom of Figure 8. 

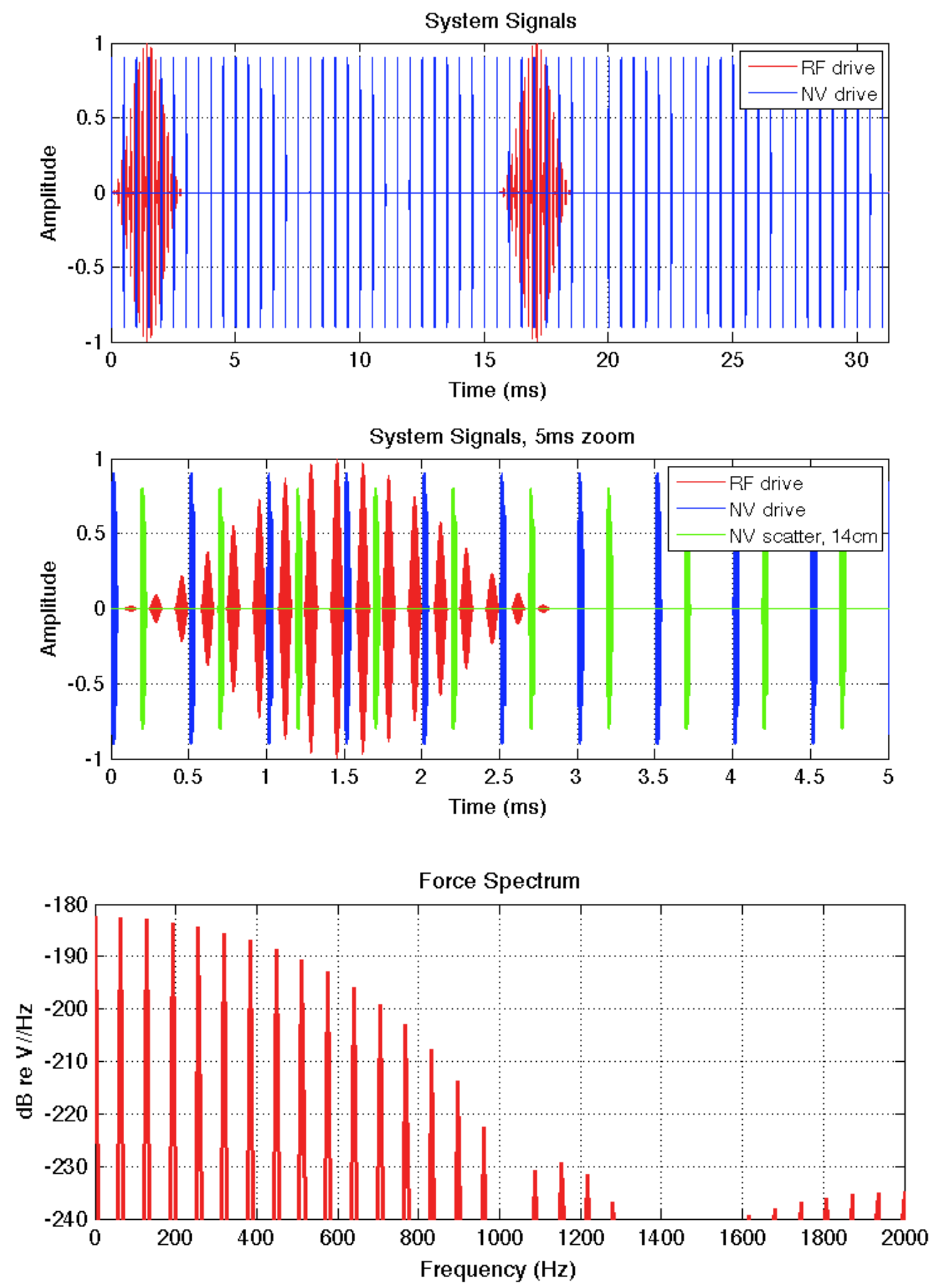

Figure 8. CFE system signals. Upper: RF and NV drive signals illustrated over two RF repetition periods (31.25ms). Middle: $R F$ and $N V$ drive signals in a shortened window (5ms), along with an illustration of $\mathrm{NV}$ backscatter from the system focal plane $(14 \mathrm{~cm})$. Lower: Low frequency spectrum of the RF force signal. 
The apparatus for CFE phantom testing is shown in Figure 9. The filters on the RF power amplifiers are intended to reduce output at drive harmonic frequencies, while the filters on the receiver are intended to minimize all RF components in the vibrometer data, and to provide improved high frequency anti-aliasing performance. The waveform generator and digitizer share a common $10 \mathrm{MHz}$ clock in order to minimize timing jitter errors. Raw vibrometer data were digitized at $10 \mathrm{MHz}$ and processed using the revised NIVMS algorithm ${ }^{\text {iii }}$ to provide axial displacement as a function of depth into the phantom.

The first tissue phantom tested with CFE was a 12" diameter, 12" tall cylinder of material produced by BluePhantom as a custom formulation to match ultrasonic properties of bottlenose dolphin tissues, as determined under a separate effort. The shear properties, determined through mechanical testing, independent of CFE, were found to in the published range of bovine muscle tissue ${ }^{\text {iv }}$. The active faces of the CFE transducers were coupled to the face of the phantom with distilled water.

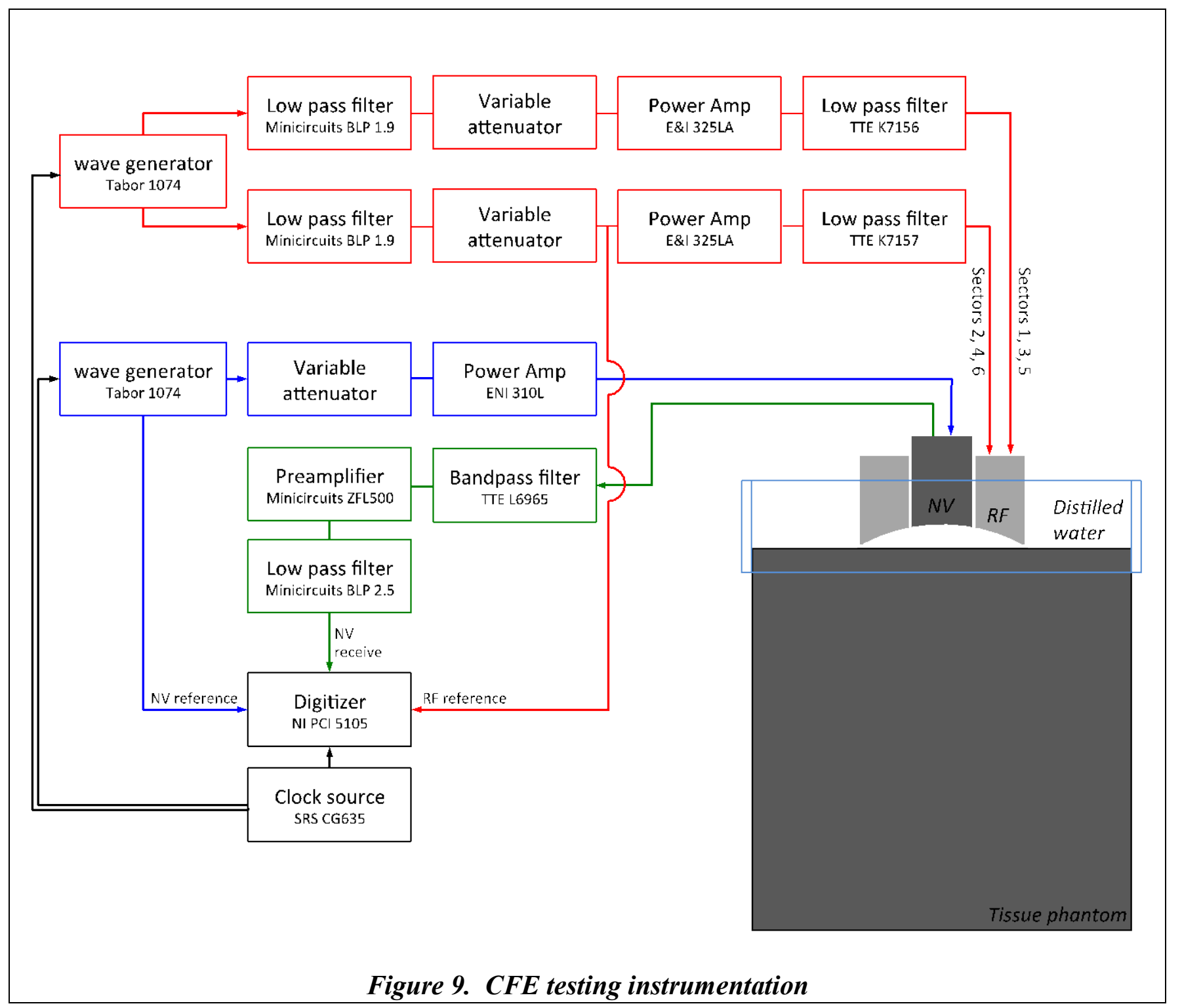


Figure 10 shows the phantom displacement spectrum obtained using an RF carrier frequency of $850 \mathrm{kHz}$, along with the relative phase of displacements generated by using carrier frequencies of 750 and $850 \mathrm{kHz}$. The use of these two carriers produces an in-situ force radius shift of $0.36 \mathrm{~mm}$. The displacement magnitudes attenuated rapidly with increasing frequency, such that based on signal to noise ratio (SNR), the useable range of frequencies for phase-based shear speed estimation was limited to $64-192 \mathrm{~Hz}$. This limitation is a direct consequence of the stiffness and viscosity of the specific phantom material and the constraint of staying within diagnostic ultrasound exposure safety limits. Softer materials (as expected with brain and fatty tissues) will produce larger displacements and phase shifts for a fixed set of transducer drive levels and carrier frequencies.

Figure 10 includes a viscoelastic model fit to magnitude and phase data. The model convolves point force viscoelastic Green's functions ${ }^{\mathrm{v}}$ with existing RF transducer intensity patterns that define the in situ force distribution as a function of frequency and space. The shear modulus is implemented in the form ${ }^{\mathrm{vi}} \mathrm{G}=\mu_{1}+\mathrm{j} \omega \mu_{2}$, where $\mu_{1}$ and $\mu_{2}$ are the shear elasticity and viscosity, respectively, and $\omega$ is angular frequency. The relationship between shear speed and modulus is $c_{s}=(G / \rho)^{1 / 2}$.

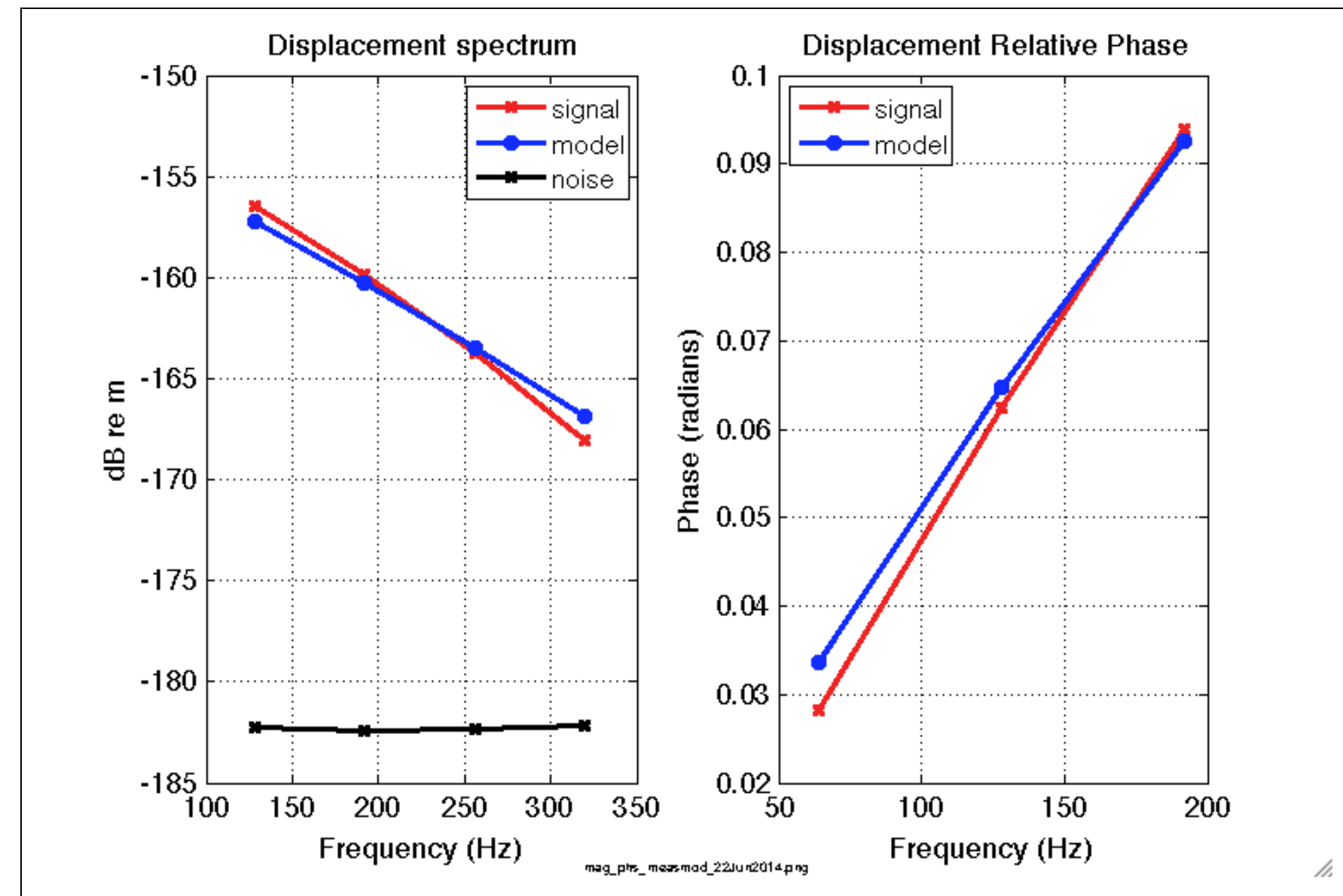

Figure 10. Measured displacement magnitude and relative phase, with viscoelastic model fit

The model fit data for the CFE experiment data are compared with values from an independent shakerbased measurement in Figure 11. For the shaker tests, the phantom was harmonically driven normal to its surface, and the resulting out of plane surface vibrations were measured with a laser vibrometer. 
Shear speed was determined from the phase difference observed as a function of distance from the drive point, and correcting for the expected difference between surface wave and shear wave speeds ${ }^{\mathrm{vii}}$. The CFE data show a shear speed approximately $20 \%$ higher than the shaker measurement, and with lower loss. Possible sources of the discrepancies are currently thought to be:

- temperature differences at the time of the CFE $\left(20^{\circ} \mathrm{C}\right)$ and shaker $\left(24^{\circ} \mathrm{C}\right)$ experiments; or

- different material sampling regions: the shaker data was sampling near-surface material, while CFE was sampling material deep in the phantom, and they may not be identical

A third, independent measurement as a function of temperature is planned with this phantom material.

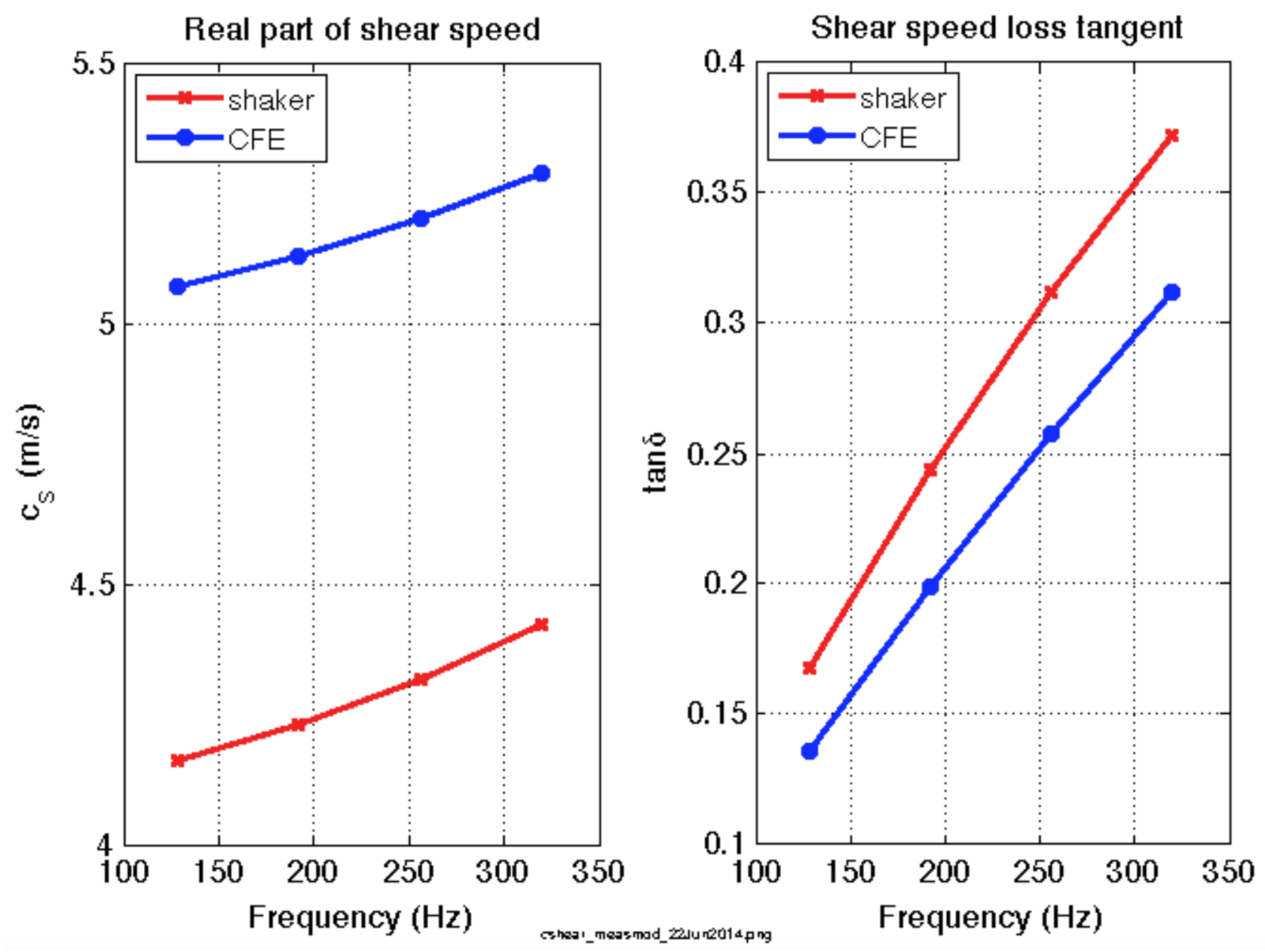

Figure 11. Phantom shear properties. Left: real part of shear speed. Right: shear speed loss factor

Work is continuing on the development of the CFE system. Specifically, testing will be conducted to:

- resolve the differences between CFE and shaker-based measurements of the original phantom; 
- exercise CFE on a phantom whose properties are in the range of published values for mammalian brain tissue;

- exercise CFE on a phantom containing inclusions having shear contrast but no echoic contrast;

- determine the ability of CFE to operate through samples of bottlenose dolphin mandibular and temporal bone (both are characterized by thin, low porosity tissue); and

- perform live animal testing as funding allows

\section{IMPACT/APPLICATIONS}

There is considerable interest in the development of structural acoustic models for the cetacean head for two main reasons: 1) to better understand biomechanics of sound reception and production in cetaceans, and 2) to understand and hopefully mitigate any harmful effects of man-made sound on their health and behavior. The development and validity of these models is severely limited by an almost complete lack of knowledge of the mechanical properties of the constituent living tissue. There is thus considerable interest in being able to measure these properties in vivo. The techniques and instrumentation investigated here should also have biomedical diagnostic application, including noninvasive examinations of stranded animals.

Testing to date has demonstrated the ability to measure shear properties of tissue-like materials at depths relevant to cetacean physiology studies, far beyond any capability previously demonstrated with an ultrasound-based system.

\section{RELATED PROJECTS}

Under award N00014-13-1-0640, the CFE system is being augmented to include techniques for determination of bulk soft tissue properties.

\section{PUBLICATIONS}

Martin, J.S., Rogers, P.H., Gray, M.D., "Pulse compression in a time variant system with application to ultrasonic vibrometry", J. Acoust. Soc. Am. (2013) 133(3): 1503-1514

Martin, J.S., Rogers, P.H., Gray, M.D., "Range discrimination in ultrasonic vibrometry: Theory and experiment”, J. Acoust. Soc. Am. (2011) 130(3): 1735-1747

\section{REFERENCES}

${ }^{\mathrm{i}}$ Sarvazyan, A., Rudenko, O, and Nyborg, W., "Biomedical applications of radiation force of ultrasound: historical roots and physical basis", Ultrasound in Med. \& Biol. 36(9): 1379-1394, 2010

ii Abbott, John G., "Rationale and derivation of MI and TI - A review", Ultrasound in Med. \& Biol. 25: 431-441, 1999

${ }^{\text {iii }}$ Martin, J.S., Rogers, P.H., Gray, M.D., "Range discrimination in ultrasonic vibrometry: Theory and experiment”, J. Acoust. Soc. Am. 130(3): 1735-1747, 2011 
${ }^{\text {iv }}$ Chen, S., Urban, M., Pislaru, C., Pinnick, R., Zheng, Y., Yao, A., Greenleaf, J., "Shearwave dispersion ultrasound vibrometry (SDUV) for measuring tissue elasticity and viscosity", IEEE Trans. Ultrason. Ferrorelectr. Freq. Control 56(1): 55-62: 2009

${ }^{v}$ Rahman, M., "Some fundamental axisymmetrical singular solutions of elastodynamics", Quarterly J. Mech. Appl. Math. 48(3): 329-342, 1995

${ }^{\text {vi }}$ Yamakoshi, Y., Sato, J., Sato, T., "Ultrasonic imaging of internal vibration of soft tissue under forced vibration", IEEE Trans. Ultrason. Ferrorelectr. Freq. Control 37(2): 45-53, 1990

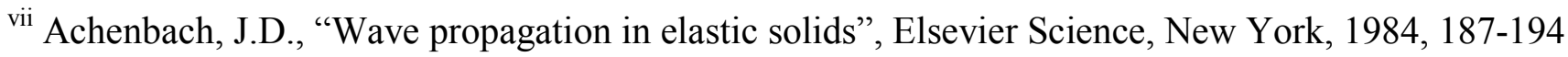

